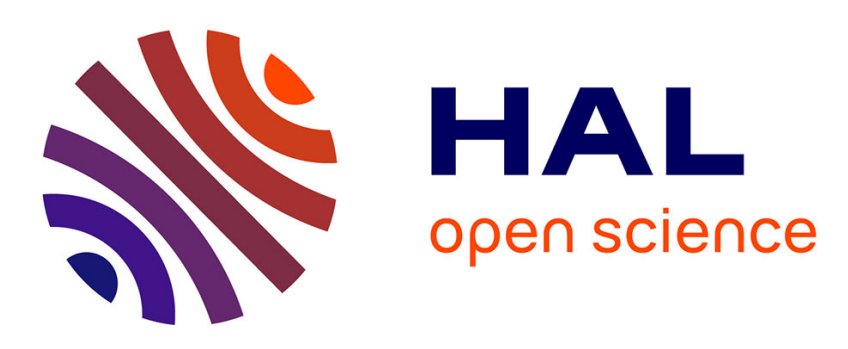

\title{
Color Adaptive Neighborhood Mathematical Morphology and its application to pixel-level classification
}

Víctor González-Castro, Johan Debayle, Jean-Charles Pinolí

\section{- To cite this version:}

Víctor González-Castro, Johan Debayle, Jean-Charles Pinolí. Color Adaptive Neighborhood Mathematical Morphology and its application to pixel-level classification. Pattern Recognition Letters, 2014, 47, pp.50 - 62. 10.1016/j.patrec.2014.01.007 . hal-01079559

\section{HAL Id: hal-01079559 https://hal.science/hal-01079559}

Submitted on 3 Nov 2014

HAL is a multi-disciplinary open access archive for the deposit and dissemination of scientific research documents, whether they are published or not. The documents may come from teaching and research institutions in France or abroad, or from public or private research centers.
L'archive ouverte pluridisciplinaire HAL, est destinée au dépôt et à la diffusion de documents scientifiques de niveau recherche, publiés ou non, émanant des établissements d'enseignement et de recherche français ou étrangers, des laboratoires publics ou privés. 


\title{
Color Adaptive Neighborhood Mathematical Morphology and its application to pixel-level classification
}

\author{
Víctor González-Castro, Johan Debayle, Jean-Charles Pinoli École Nationale Supérieure des Mines de Saint-Étienne \\ LGF UMR CNRS 5307 \\ 42023 Saint-Etienne Cedex 2, FRANCE \\ Email: victor.gonzalez@emse.fr, debayle@emse.fr,pinoli@emse.fr
}

\begin{abstract}
In this paper spatially adaptive Mathematical Morphology (MM) is studied for color images. More precisely, the General Adaptive Neighborhood Image Processing (GANIP) approach is generalized to color images. The basic principle is to define a set of locally Color Adaptive Neighborhoods (CAN), one for each point of the image, and to use them as adaptive structuring elements (ASE) for morphological operations. These operators have been applied to images in different color spaces and compared them with other kinds of ASEs extended to color images. Results show that the proposed method is more respectful with the borders of the objects, as well as with the color transitions within the image. Finally, the proposed adaptive morphological operators are applied to the classification of color texture images.
\end{abstract}

\section{INTRODUCTION}

Mathematical Morphology (MM) is a theory for image analysis based on set theory. It has been developed from the ideas of G. Matheron (Matheron, 1975) and J. Serra (Serra, 1982) in the École des Mines de Paris. Initially, they established its basis focusing on binary images, but it was later extended to gray level images (Sternberg, 1986). Afterwards, Haralick et al. reviewed morphological operators, as well as their relations and properties (Haralick et al., 1987).

Mathematical morphology has been successfully used in several applications: remote sensing (Örsan Aytekin and lkay Ulusoy, 2011), biomedicine (Bouraoui et al., 2010), quality control in industry (Priya et al., 2011), geoscience (Tuia et al., 2009) or texture description (González-Castro et al., 2012) are just some examples.

For some applications, a drawback in Mathematical morphology is the fact that the structuring element (SE) used in morphological operations classically have a fixed shape and size, which has serious disadvantages such as creating artificial patterns or removing significant details. In addition, it is not straightforward to set the shape of the SE that best suits certain image structures, as some authors have discussed (de Ves et al., 2006). Huet and Mattioli presented a method to generate a minimal set of SEs that left the texture invariant and used them to carry out some morphological transformations for texture defect detection (Huet and Mattioli, 1996). Asano and his co-workers (Asano et al., 2003) claimed that the best SE to compute the pattern spectrum of a texture would be the one whose shape is the most similar to the granules within it, so they selected the one which reduced the variance of the size distribution.

This kind of selection of SEs makes necessary to have a priori information about the images to be processed (e.g. size and orientation of structures within the images). This is a serious shortcoming, since such knowledge is not always available in real computer vision tasks.

Some works deal with the computation of structuring elements that adapt themselves to the local features of the image at each pixel. Shih and Cheng presented an adaptive edge-linking method based on MM which used an elliptical SE whose orientation and size were adjusted to some local features of the image (Shih and Cheng, 2004). Landström and Thurley (Landström and Thurley, 2013) proposed a framework for adaptive morphology where elliptical structuring elements ranged from lines to disks, and varied their orientation within the data by capturing the eigenvalues and eigenvectors of the Local Tensor Structure. Bouaynaya and her co-workers presented an approach for image restoration and skeletonization which used spatial-variant mathematical morphology (Bouaynaya et al., 2006). The reader interested in more details is referred to (Bouaynaya et al., 2008) and (Bouaynaya and Schonfeld, 2008).

Lerallut et al. introduced the concept of morphological amoebas, which are adaptive structuring elements whose shape is locally adapted to the image contour variations, by means of a weighted geodesic distance (Lerallut et al., 2007). Grazzini and Soille also proposed a filtering approach which used mathematical morphology aiming at edge-preserving smoothing which also used structuring elements obtained by means of a geodesic distance criterion (Grazzini and Soille, 2009). Likewise, González-Castro et al. used combined geodesic and Euclidean distance criteria to compute the SE at each point of the image, in this case for classification purposes, characterizing the textures by means of adaptive pattern spectra (González-Castro et al., 2012). Recently, Curić et al. presented salience adaptive structuring elements, that vary both their shape and size according to the salience of edges in the image. They are less flexible than morphological amoebas but their shape is less affected by noise (Ćurić et al., 2012).

Debayle and Pinoli presented the so-called General Adaptive 
Neighborhood Image Processing (GANIP) approach from a theoretical and practical point of view in (Debayle and Pinoli, 2006a) and (Debayle and Pinoli, 2006b), respectively. Within this paradigm a General Adaptive Neighborhood (GAN) is constituted by connected components whose point intensity values - measured in relation to a selected criterion such as luminance, contrast, thickness, etc. - fit within a specified range of homogeneity tolerance. These GANs are used as adaptive structuring elements in morphological operations.

As it has been pointed out, morphological operators were initially defined - and, therefore, used - for binary and grayscale images. These operators need a total order relationship. The extension of mathematical morphology theory to color images is not straightforward as, due to the vectorial nature of color data, there is not such notion. Jesus Angulo studied such morphological color operators and proposed some total orderings for color images (Angulo, 2007). A comprehensive survey on different approches to multivariate MM can be found in (Aptoula and Lefvre, 2007).

Adaptive MM for color images has been few investigated. Morphological Amoebas (Lerallut et al., 2007) and the approach introduced by Grazzini and Soille (Grazzini and Soille, 2009) were extended to multichannel images with filtering purposes. However, they were not used as structuring elements in color adaptive mathematical morphology.

Debayle and Pinoli extended their GANIP framework to color images by defining Color Adaptive Neighborhoods (CAN) and morphological operations using the lexicographical ordering as total order relationship (Debayle and Pinoli, 2014).

In this paper CAN-based morphological operators are defined and studied with a different total order relationship. Finally, these color adaptive morphological operators are used for defining descriptors at pixel-level for classification purposes.

\section{General Adaptive Neighborhood Image PROCESSING (GANIP)}

In this section a review of the so-called General Adaptive Neighborhood Image Processing (GANIP) framework, introduced by Debayle and Pinoli (Debayle and Pinoli, 2006a), will be made.

\section{A. An overview on the framework}

In the GANIP approach a General Adaptive Neighborhood (GAN) is defined for each point of the image to be analyzed. A GAN is a subset of the spatial support $D$ constituted by connected points whose values in relation to a selected criterion (luminance, contrast, ...) fit within a homogeneity tolerance.

Let $I$ be the set of gray-level images defined on the spatial support $D \subseteq \mathbb{R}^{2}$ and valued in an interval $\tilde{E} \subseteq \mathbb{R}$. Thus, $I=\{f \mid \bar{f}: D \rightarrow \tilde{E}\}$. Let $f \in I$ be an image, and $f_{0} \in I$ be the so-called pilot or reference image. For each point $x \in D$ belonging to $f$, the GANs (denoted $V_{m}^{f_{0}}(x)$ ) are subsets in $D$ built upon $f_{0}$ (a criterion mapping based on local measurements such as luminance, contrast, etc.) in relation to a homogeneity tolerance $m \in \mathbb{R}^{+}$. More precisely, $V_{m}^{f_{0}}(x)$ has to fulfill two conditions:
- The criterion measurement of its points is close to the one of $x$

$$
\forall y \in V_{m}^{f_{0}}(x): \quad\left|f_{0}(y)-f_{0}(x)\right| \leq m
$$

- The GAN is a path-connected set (according to the usual Euclidean topology on $D \subseteq \mathbb{R}^{2}$ )

Thus, the GANs are formally defined as:

$\forall\left(m, f_{0}, x\right) \in \mathbb{R}^{+} \times I \times D \quad V_{m}^{f_{0}}(x)=C_{\left\{y \in D ;\left|f_{0}(y)-f_{0}(x)\right| \leq m\right\}}(x)$

where $C_{X}(x)$ denotes the path connected component of $X \subseteq D$ containing $x \in D$. Therefore, it is ensured that $\forall x \in D \quad x \in V_{m}^{f_{0}}(x)$.

However, these GANs do not satisfy the symmetry property, defined as:

$$
\forall(x, y) \in D^{2} \quad y \in A(x) \Longleftrightarrow x \in A(y)
$$

where $\{A(x)\}_{x \in D}$ is a collection of subsets $A(x) \subseteq D$. For this reason, GANs defined in equation (1) are called Weak General Adaptive Neighborhoods (W-GANs). From a visual point of view, the symmetry property is closely linked to the human visual perception. Moreover, the notion of symmetry is topologically relevant (Debayle and Pinoli, 2006a).

In order to get this property satisfied, a new set of GANs, called Strong General Adaptive Neighborhoods (S-GANs) is defined as:

$$
\forall\left(m, f_{0}, x\right) \in \mathbb{R}^{+} \times I \times D \quad N_{m}^{f_{0}}(x)=\bigcup_{z \in D}\left\{V_{m}^{f_{0}}(z) \mid x \in V_{m}^{f_{0}}(z)\right\}
$$

The reader interested in further theoretical aspects on GANs is referred to (Debayle and Pinoli, 2006a).

\section{B. Application to Mathematical Morphology}

The two fundamental operators in Mathematical Morphology are erosion and dilation which are defined respectively as:

$$
\begin{aligned}
& E_{B}(f(x))=\inf \{f(w): w \in B(x)\} \\
& D_{B}(f(x))=\sup \{f(w): w \in \check{B}(x)\}
\end{aligned}
$$

where $B(x)$ denotes the structuring element $B$ whose origin is located at point $x$, and $\check{B}$ stands for its reflected subset, defined as

$$
\check{B}(x)=\{z \mid x \in B(z)\} .
$$

The idea behind the Adaptive Neighborhood Mathematical Morphology is to substitute the usual structuring elements by the adaptive ones at each pixel of the image.

In the particular case of the GANIP approach, the symmetric S-GANs are used. Therefore GAN-based adaptive erosion and dilation can be expressed as:

$$
E_{m}^{f_{0}}(f)(x)=\inf _{w \in N_{m}^{f_{0}}(x)}(f(w))
$$




$$
D_{m}^{f_{0}}(f)(x)=\sup _{w \in N_{m}^{f_{0}}(x)}(f(w))
$$

Thereafter, more advanced GAN morphological operators can be defined (Pinoli and Debayle, 2009).

\section{COLOR MORPHOLOGY}

In this section color spaces, color orderings and finally color mathematical morphology will be addressed.

As it has been pointed out in section II-B, morphological operators need the definition of a total order relationship between the points to be processed. As points in gray-level images are valued in $\mathbb{R}$, there is a natural notion of total order. However, there is not such straightforward notion in vector spaces. Therefore, it is difficult to order data in color images, as each point is a vector of $n$ components (with, generally, $n=3)$.

In addition, the colors in digital images can be represented in different color spaces (Joblove and Greenberg, 1978) and the choice of one or other may have influence in the final results (Busin et al., 2008; Yang et al., 2010).

\section{A. Color spaces and distances}

The most straightforward way to represent and manipulate digital color images is using the RGB color space. However, it has some drawbacks, such as the correlation of its components or lack of clear human interpretation (Palus, 1998). There are some color representations that have been created to be more perceptually intuitive, such as the $\mathrm{L}^{*} \mathrm{a} * \mathrm{~b} *$ or polar color spaces (i.e., HSV, HSL, HSI, etc.) (van den Broek et al., 2008). In this paper, the the color adaptive morphological operators have been applied on the RGB, L*a*b* and HSL color spaces. The equations for making the transformations among them are well known (Palus, 1998; Joblove and Greenberg, 1978), hence, they are not included.

Let us define the notation for color images, as it was made in section II-A. Let $\tilde{E}_{A}, \tilde{E}_{B}$ and $\tilde{E}_{C}$ three sets of scalar values (specifically, subsets of $\mathbb{R}$ ), and let $I_{C}=\left\{f \mid f: D \rightarrow \tilde{E}_{A B C}\right\}$ be the set of color images defined in the spatial support $D$ and valued in the three-dimensional vector space $\tilde{E}_{A B C}=$ $\tilde{E}_{A} \times \tilde{E}_{B} \times \tilde{E}_{C}$. In this notation, $A, B$ and $C$ stand for the color components of the color space that is used (e.g. $c_{i} \in$ $\tilde{E}_{L * a * b *} \Longleftrightarrow c_{i}=\left\{\left(L *_{i}, a *_{i}, b *_{i}\right) ; L *_{i} \in \tilde{E}_{L *}, a *_{i} \in\right.$ $\left.\left.\tilde{E}_{a *}, b *_{i} \in \tilde{E}_{b *}\right\}\right)$. The elements of $I_{C}$ are denoted as $f$, so that the value of $f$ at $x \in D$ in the color space $A B C$ is denoted by $f(x)=\left\{f_{A}(x), f_{B}(x), f_{C}(x)\right\}$.

Note that the sets $\tilde{E}_{R}, \tilde{E}_{G}$ and $\tilde{E}_{B}$, corresponding to the color components of the channels Red, Green and Blue in the RGB representation, as well as the sets $\tilde{E}_{L *}, \tilde{E}_{a *}$ and $\tilde{E}_{b *}$ - $\mathrm{L}^{*} \mathrm{a}^{*} \mathrm{~b}^{*}$ - and $\tilde{E}_{S}$ and $\tilde{E}_{L}$ corresponding to the saturation and luminance in the HSL color space are totally ordered sets. However, as the hue component is an angular function defined on the unit circle $\mathscr{C}\left(E_{H}=\mathscr{C}\right)$, it is not fitted with a total ordering. Let $\left(h_{i}, h_{j}\right) \in \mathscr{C} \times \mathscr{C}$, the angular difference (Hanbury and Serra, 2001) is defined as:

$$
h_{i} \div h_{j}= \begin{cases}\left|h_{i}-h_{j}\right| & \text { if }\left|h_{i}-h_{j}\right| \leq 180^{\circ} \\ 360^{\circ}-\left|h_{i}-h_{j}\right| & \text { if }\left|h_{i}-h_{j}\right|>180^{\circ}\end{cases}
$$

Therefore, fixing an origin of the hues, $h_{0}$, a $h_{0}$-centered hue function can be defined as $f_{H}(x) \div h_{0}$ (or, making an abuse of notation, $\left(f_{H} \div h_{0}\right)(x)$ ).

Now, it is possible to define a distance measure between colors in the different spaces. Let $c_{i}$ and $c_{j}$ be two colors. In the case of the RGB color space, the Euclidean distance can be used:

$$
d_{R G B}\left(c_{i}, c_{j}\right)=\sqrt{\left(c_{i}^{R}-c_{j}^{R}\right)^{2}+\left(c_{i}^{G}-c_{j}^{G}\right)^{2}+\left(c_{i}^{B}-c_{j}^{B}\right)^{2}}
$$

The Euclidean distance in the CIE $\mathrm{L}^{*} \mathrm{a} * \mathrm{~b} *$ color space, denoted by $\Delta E$,

$$
\Delta E=\sqrt{\left(c_{i}^{L *}-c_{j}^{L *}\right)^{2}+\left(c_{i}^{a *}-c_{j}^{a *}\right)^{2}+\left(c_{i}^{b *}-c_{j}^{b *}\right)^{2}},
$$

is particularly used in the literature, although other other distances for this color space, such as the $\Delta E_{94}$ (CIE, 1995) or the $\Delta E_{2000}$ (CIE, 2001), have been proposed. Therefore, in this article $d_{L * a * b *}\left(c_{i}, c_{j}\right) \equiv \Delta E$.

There are some proposals in (Angulo, 2007) for color distances in the HSL space. The most appropriate among them seems to be:

$d_{H S L}\left(c_{i}, c_{j}\right)=\sqrt{\left(c_{i}^{L}-c_{j}^{L}\right)^{2}+\left(c_{i}^{S}\right)^{2}+\left(c_{j}^{S}\right)^{2}-2 c_{i}^{S} c_{j}^{S} \cos \left(c_{i}^{H} \div c_{j}^{H}\right)}$

as colors are expressed in cylindrical coordinates in this space.

\section{B. Color ordering relations and morphological operations}

Morphological operators need the sets of the intensities to be processed to hold a total order relationship. However, in the case of color images this is not straightforward due to the metric nature of their points.

In the literature several order relationships have been proposed (i.e., marginal, lexicographical, partial or reduced ordering) (Barnett, 1976; Angulo, 2007). Let $c_{1}=\left(c_{1}^{A}, c_{1}^{B}, c_{1}^{C}\right)$ and $c_{2}=\left(c_{2}^{A}, c_{2}^{B}, c_{2}^{C}\right)$ be two colors which belong to $E_{A B C}$. The lexicographical order on the color space $A B C$ with the component ordering $A \rightarrow B \rightarrow C$, denoted $\prec_{L e x}$, is defined as:

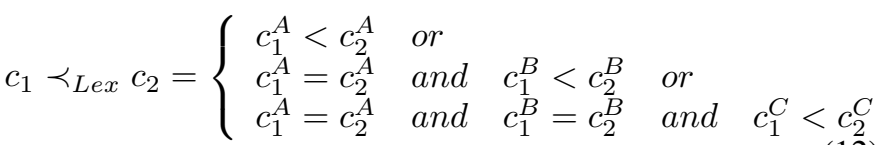

The reduced ordering with respect to a reference color $c_{0}$, denoted by $\prec_{c_{0}}$, between two points claims that

$$
c_{1} \prec_{c_{0}} c_{2} \Longleftrightarrow d_{A B C}\left(c_{1}, c_{0}\right)>d_{A B C}\left(c_{2}, c_{0}\right)
$$

where $d_{A B C}(\cdot, \cdot)$ represents the suitable distance between two points in the color space $A B C$ (see section III-A). It is easy to demonstrate that this is a partial ordering. Intuitively, it can be 
seen that two different colors may be equidistant to a reference color - e.g. in RGB, if $c_{0}=(0,0,0), c_{1}=(255,255,0)$ and $c_{2}=(255,0,255)$ then $d_{R G B}\left(c_{1}, c_{0}\right)=d_{R G B}\left(c_{2}, c_{0}\right)-$.

A combination of both has already been proposed in (Angulo, 2007), called $\Omega$-ordering, denoted as $\prec_{\Omega}$ and shown in equation (14).

$c_{1} \prec{ }_{\Omega} c_{2}=\left\{\begin{array}{l}d_{A B C}\left(c_{1}, c_{0}\right)>d_{A B C}\left(c_{2}, c_{0}\right) \text { or } \\ d_{A B C}\left(c_{1}, c_{0}\right)=d_{A B C}\left(c_{2}, c_{0}\right) \text { and } \\ \left\{\begin{array}{l}c_{1}^{A}<c_{2}^{A} \text { or } \\ c_{1}^{A}=c_{2}^{A} \text { and } c_{1}^{B}<c_{2}^{B} \text { or } \\ c_{1}^{A}=c_{2}^{A} \text { and } c_{1}^{B}=c_{2}^{B} \text { and } c_{1}^{C}<c_{2}^{C}\end{array}\right.\end{array}\right.$

The resulting color erosion and dilation of an image $\mathbf{f} \in I_{C}$ at point $x \in D$ by means of the structuring element $B \subseteq D$ is given respectively by:

$$
\begin{aligned}
& E_{*, B}(f)(x)=\inf _{*}\{f(w): w \in B(x)\} \\
& D_{*, B}(f)(x)=\sup _{*}\{f(w): w \in \check{B}(x)\}
\end{aligned}
$$

where $\sup _{*}$ and $\inf _{*}$ stands for the supremum and infimum according to the total order relationship * - e.g., lexicographical, $\Omega$-ordering -.

In relation to the total order relationships that were presented before, the lexicographical one holds some disadvantages. Actually, it is not clear how to decide which is the color component with more priority (i.e., which one is more important), specially in color spaces in which two or more components contain chromatic information (i.e., RGB or $\mathrm{L}^{*} \mathrm{a}^{*} \mathrm{~b}$ ). In addition, results of operations which need a total ordering relationship would be clearly influenced by this dominant color component. Anyway, some approaches have been introduced in the literature to limit this excessive influence given by the priority color component (Aptoula and Lefvre, 2008).

On the other hand, $\Omega$-ordering seems more fair, as it does not make operations to be influenced by any color component (except in the few cases where $d_{A B C}\left(c_{1}, c_{0}\right)=d_{A B C}\left(c_{2}, c_{0}\right)$ ).

It is true that the $\Omega$-ordering is influenced by the choice of reference color $c_{0}$, but it is more straightforward to set $c_{0}$ than deciding which color component is more important. Actually, regarding the definition in (14) it can be concluded that the upper bound is $c_{0}$ itself thus, for example, it seems more intuitive to set $c_{0}=(255,255,255)$ as the "biggest color" in the RGB color space than setting $\mathrm{R}, \mathrm{G}$ or $\mathrm{B}$ as the most important color component. Therefore, the $\Omega$-ordering is used in all the operations in the rest of the paper.

\section{CAN-BASEd MathematicAl Morphology}

\section{A. Color Adaptive Neighborhoods (CAN)}

1) CAN Definition: Let $x$ be a point of the spatial support $D$, and $f_{0} \in I_{C}$ a color image called the pilot image. The Color Adaptive Neighborhoods (CANs), denoted $V_{m}^{f_{0}}(x)$, are built upon $f_{0}$ in relation with a homogeneity tolerance value $m \in \mathbb{R}^{+}$. The CANs must fulfill the same two conditions as the GANs (see section II-A), thus, for any CAN:
- its points must have a color value close to the one of $x$, and

- the set has to be path-connected, considering the usual Euclidean topology on $D \subseteq \mathbb{R}^{2}$.

Therefore, CANs are formally defined as:

$$
V_{m}^{f_{0}}(x)=C_{\left\{y \in D ; d_{\gamma}\left(f_{0}(y), f_{0}(x)\right) \leq m\right\}}(x)
$$

where $C_{X}(x)$ denotes the path-connected component of $X$ which contains $x \in D$, and $d_{\gamma}\left(f_{0}(y), f_{0}(x)\right)$ stands for the distance between the color points $f_{0}(y)$ and $f_{0}(x)$ in the color space $\gamma$. The figure 1 illustrates the CANs of three selected points in an image.

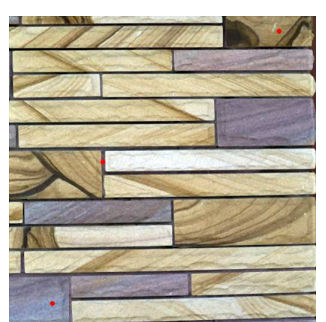

(a)

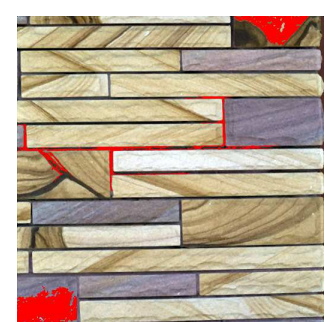

(b)
Fig. 1. The CANs (right) of three points (left) of a color image $f$ computed in the RGB color space with a tolerance parameter $m=40$.

2) CANs properties: Color Adaptive Neighborhoods satisfy several properties:

1) Reflexivity:

$$
\forall x \in D: \quad x \in V_{m}^{f_{0}}(x)
$$

Proof: $\forall x \in D$ and $\forall m \in \mathbb{R}^{+}$: $d_{\gamma}\left(f_{0}(x), f_{0}(x)\right)=0 \leq m$

2) Increasing with respect to $m$ :

Let $\left(m_{1}, m_{2}\right) \in \mathbb{R} \times \mathbb{R}$. Then:

$$
m_{1} \leq m_{2} \Longrightarrow V_{m_{1}}^{f_{0}}(x) \subseteq V_{m_{2}}^{f_{0}}(x)
$$

Proof: Let $P_{x}^{y}$ be a path between $x$ and $y$. So,

$$
\begin{aligned}
y \in V_{m_{1}}^{f_{0}}(x) & \Longrightarrow \exists P_{x}^{y}: d_{\gamma}\left(f_{0}(x), f_{0}(y)\right) \leq m_{1} \\
& \Longrightarrow \exists P_{x}^{y}: d_{\gamma}\left(f_{0}(x), f_{0}(y)\right) \leq m_{2} \\
& \Longrightarrow y \in V_{m_{2}}^{f_{0}}(x)
\end{aligned}
$$

3) Equality between iso-valued points:

Let $(x, y) \in D \times D$ with $x \in V_{m}^{f_{0}}(y)$ and $f_{0}(x)=$ $f_{0}(y)$. Then:

$$
V_{m}^{f_{0}}(x)=V_{m}^{f_{0}}(y)
$$

Proof: Let $z$ be a point in $V_{m}^{f_{0}}(x)$. There exists a path $P_{x}^{z}$ such that $\forall t \in P_{x}^{z}: d_{\gamma}\left(f_{0}(x), f_{0}(t)\right) \leq m$. In addition, $x \in V_{m}^{f_{0}}(y)$, so there exists a path $P_{y}^{x}$ such that $\forall u \in P_{y}^{x}: d_{\gamma}\left(f_{0}(y), f_{0}(u)\right) \leq m$.

Let be $P_{y}^{z}=P_{y}^{x} \cup P_{x}^{z}$, and let $u \in P_{y}^{z}$. There are two possibilities: 
$v \in P_{x}^{z} \quad \Longrightarrow \quad d_{\gamma}\left(f_{0}(x), f_{0}(v)\right) \quad=$ $d_{\gamma}\left(f_{0}(y), f_{0}(v)\right) \leq m$

$v \in P_{y}^{x} \Longrightarrow d_{\gamma}\left(f_{0}(y), f_{0}(v)\right) \leq m$

Anyway, $\forall v \in P_{y}^{z}: d_{\gamma}\left(f_{0}(y), f_{0}(v)\right) \leq m \Longrightarrow z \in$ $V_{m}^{f_{0}}(y)$. Therefore, $V_{m}^{f_{0}}(x) \subseteq V_{m}^{f_{0}}(y)$.

Making a similar reasoning, and considering that $\forall w \in$ $P_{a}^{b}: w \in P_{b}^{a}$, it can be proved that $V_{m}^{f_{0}}(y) \subseteq V_{m}^{f_{0}}(x)$ and, therefore, that $V_{m}^{f_{0}}(x)=V_{m}^{f_{0}}(y)$

\section{B. CAN Morphology}

The idea behind the Color Adaptive Neighborhood Mathematical Morphology, as well as in the case of the GAN morphology (section II-B), is to replace the classic Structuring Elements by CANs. However, if they were used directly as Adaptive Structuring Elements (ASEs), the symmetry property $\left(x \in V_{m}^{f_{0}}(y) \Longleftrightarrow y \in V_{m}^{f_{0}}(x)\right.$ ) would not be satisfied. Therefore, the color equivalent to the S-GANs are used as ASEs:

$$
N_{m}^{f_{0}}(x)=\bigcup_{z \in D}\left\{V_{m}^{f_{0}}(z) \mid x \in V_{m}^{f_{0}}(z)\right\}
$$

The symmetry property is relevant for visual, topological, morphological and practical reasons (Debayle and Pinoli, 2006a).

Thus, the elementary adaptive morphological operators are defined as:

$$
\begin{gathered}
E_{m}^{f_{0}}(f)(x)=\inf _{\delta}\left\{f(w): w \in N_{m}^{f_{0}}(x)\right\} \\
\left.D_{m}^{f_{0}}(f)(x)=\sup _{\delta}\left\{f(w): w \in N_{m}^{f_{0}}(x)\right)\right\}
\end{gathered}
$$

where $\inf _{\delta}$ and $\sup _{\delta}$ denote the infimum and supremum in terms of the total order relationship $\prec_{\delta}$.

It is important to remark the need to compute the ASEs from the pilot image $f_{0}$ (Lerallut et al., 2007), so that they will have the same shape in successive runs. It makes possible to properly define adaptive openings and closings:

$$
\begin{aligned}
& O_{m}^{f_{0}}(f)=D_{m}^{f_{0}}\left(E_{m}^{f_{0}}(f)\right) \\
& C_{m}^{f_{0}}(f)=E_{m}^{f_{0}}\left(D_{m}^{f_{0}}(f)\right)
\end{aligned}
$$

Moreover, it is possible to further combine dilations and erosions and define other advanced operations (e.g. alternate filters, toggle contrast, top hat, etc.).

1) Properties: Both erosion and dilation operators, using CANs computed on the pilot image $f_{0}$, define input-adaptive adjunct morphological operators, i.e. satisfying the following equivalence:

Let $m \in \mathbb{R}$ and $\left(f_{0}, f, g\right) \in I_{C}{ }^{3}$. Then:

$$
D_{m}^{f_{0}}(f) \prec_{\delta} g \Longleftrightarrow g \prec_{\delta} E_{m}^{f_{0}}(g)
$$

Proof:

$$
\begin{aligned}
D_{m}^{f_{0}}(f) \prec_{\delta} g & \Longleftrightarrow D_{m}^{f_{0}}(f)(x) \prec_{\delta} g(x), \forall x \in D \\
& \left.\Longleftrightarrow \sup _{\delta}\left\{f(w): w \in N_{m}^{f_{0}}(x)\right)\right\} \prec_{\delta} g(x), \forall x \in D \\
& \Longleftrightarrow f(w) \prec_{\delta} g(x), \forall x \in D, \forall w \in N_{m}^{f_{0}}(x) \\
& \Longleftrightarrow f(w) \prec_{\delta} g(x), \forall w \in D, \forall x \in N_{m}^{f_{0}}(w) \\
& \Longleftrightarrow f(w) \prec_{\delta} \inf _{\delta}\left\{g(x): x \in N_{m}^{f_{0}}(w)\right\}, \forall w \in D \\
& \Longleftrightarrow f(w) \prec_{\delta} E_{m}^{f_{0}}(g)(w), \forall w \in D \\
& \Longleftrightarrow f \prec_{\delta} E_{m}^{f_{0}}(g)
\end{aligned}
$$

In addition, CAN-based morphological operators satisfy these other properties:

1) Increasing:

$$
f \prec_{\delta} g \Longrightarrow\left\{\begin{array}{l}
D_{m}^{f_{0}}(f) \prec_{\delta} D_{m}^{f_{0}}(g) \\
E_{m}^{f_{0}}(f) \prec_{\delta} E_{m}^{f_{0}}(g) \\
O_{m}^{f_{0}}(f) \prec_{\delta} O_{m}^{f_{0}}(g) \\
C_{m}^{f_{0}}(f) \prec_{\delta} C_{m}^{f_{0}}(g)
\end{array}\right.
$$

2) Extensiveness

$$
O_{m}^{f_{0}}(f) \prec_{\delta} f \prec_{\delta} C_{m}^{f_{0}}(f)
$$

3) Distributivity with $\sup _{\delta}, \inf _{\delta}$

$$
\forall f_{i} \in I_{C}^{n}: \quad\left\{\begin{array}{l}
\inf _{\delta}\left\{E_{m}^{f_{0}}\left(f_{i}\right)\right\}=E_{m}^{f_{0}}\left(\inf _{\delta}\left\{f_{i}\right\}\right) \\
\sup _{\delta}\left\{D_{m}^{f_{0}}\left(f_{i}\right)\right\}=D_{m}^{f_{0}}\left(\sup _{\delta}\left\{f_{i}\right\}\right)
\end{array}\right.
$$

4) Idempotence

$$
\left\{\begin{array}{l}
C_{m}^{f_{0}} \circ C_{m}^{f_{0}}(f)=C_{m}^{f_{0}}(f) \\
O_{m}^{f_{0}} \circ O_{m}^{f_{0}}(f)=O_{m}^{f_{0}}(f)
\end{array}\right.
$$

5) Increasing, decreasing with respect to $m$ $\forall\left(m_{1}, m_{2}\right) \in \mathbb{R}^{+} \times \mathbb{R}^{+}:$

$$
m_{1} \leq m_{2} \Longrightarrow\left\{\begin{array}{l}
D_{m_{1}}^{f_{0}}(f) \prec_{\delta} D_{m_{2}}^{f_{0}}(f) \\
E_{m_{2}}^{f_{0}}(f) \prec_{\delta} E_{m_{1}}^{f_{0}}(f)
\end{array}\right.
$$

The proofs for these properties are not given, as they are inferred from the adjunction property given by (26), the lattice theory and the properties of the CANs (section IV-A2). The interested reader is addressed to (Debayle and Pinoli, 2006a), where similar proofs are given for GAN-based gray-level morphology.

\section{Other adaptive Structuring Elements}

The behavior of Color Adaptive Neighborhoods will be compared with other adaptive SEs defined in the literature and their application to morphological operations (as it was explained in section IV-B). These operators are the Morphological Amoebas, defined by Lerallut et al. (Lerallut et al., 2007), and the "Adaptive Geodesic Neighborhoods" defined by Grazzini and Soille (Grazzini and Soille, 2009). 
First of all, the way how they are defined for gray-level images, and how they have been used in color images will be summarize. Thereafter some illustrations will be given to see how morphological operators work with each of them.

A gray-level image $f$ can also be represented by a surface $S$ with two spatial coordinates in $D$ and other coordinate corresponding to the gray level value of the image at these spatial coordinates. A geodesic distance between two points $(x, f(x))$ and $(y, f(y))$ on $S$ is the minimum cost to travel from one to the other along the surface. Since digital images are being considered, discrete paths have been used (continuous ones are not suitable).

Let $P_{x y}$ be a geodesic path connecting $x$ and $y$. It can be considered as a set $\left\{x_{1}, x_{2}, \ldots, x_{n+1}\right\}$, where $x_{1}=x$ and $x_{n}=$ $y$. The cost of the geodesic path connecting two adjacent points $x_{i}$ and $x_{i+1}$ (with $i \in[1, n]$ ) must take into account both (a) the spatial distance between them and (b) the distance between their corresponding gray-level values $f\left(x_{i}\right)$ and $f\left(x_{i+1}\right)$. The above mentioned spatial distance can be estimated by means of any distance measure - Euclidean, Manhattan, Chessboard, a weighted distance like the $\langle 3,4\rangle$ distance, etc. -. The cost, $C$, of the path $P_{x y}$ is

$$
C\left(P_{x y}\right)=\sum_{i=1}^{n} c\left(x_{i}, x_{i+1}\right)
$$

and the geodesic distance between $x$ and $y$ is

$$
d(x, y)=\min _{P_{x y}} C\left(P_{x y}\right)
$$

1) Morphological Amoebas: As it is defined in (Lerallut et al., 2007), a morphological amoeba centered in $x \in D$ is:

$$
A_{r}(x)=\{y \in D: d(x, y)<r\}
$$

where $r$ is the so-called radius of the amoeba.

For morphological amoebas the cost between two adjacent points $x_{i}, x_{i+1}$ in $P_{x y}$ is defined by:

$$
c\left(x_{i}, x_{i+1}\right)=\left\|x_{i}-x_{i+1}\right\|_{\mathbb{R}^{2}}+\lambda\left|f\left(x_{i}\right)-f\left(x_{i+1}\right)\right|
$$

where $\lambda>0$

In this work the considered spatial distance $\left\|x_{i}-x_{i+1}\right\|$ is the $\langle 3,4\rangle$ distance, and the $\lambda$ parameter has been fixed to 0.25 .

2) Adaptive Geodesic Neighborhoods: Grazzini and Soille use the same principle for their locally Adaptive Geodesic Neighborhoods (Grazzini and Soille, 2009), which will be called AGN in this paper. The cost $c\left(x_{i}, x_{i+1}\right)$ between two adjacent pixels $x_{i}$ and $x_{i+1}$, called $\Delta$-time, is defined as:

$$
c\left(x_{i}, x_{i+1}\right)=\frac{1}{2}\left|f\left(x_{i}\right)-f\left(x_{i+1}\right)\right| \cdot\left\|x_{i}-x_{i+1}\right\|_{\mathbb{R}^{2}}
$$

As the work presented in this paper deals with with color images, the distance $d_{A B C}\left(f\left(x_{i}\right), f\left(x_{i+1}\right)\right)$ for color space $A B C$ should be used instead of the term $\left|f\left(x_{i}\right)-f\left(x_{i+1}\right)\right|$ in both equations (33) and (34).

Following the idea stated in section IV-B, the classic Structuring Elements will be replaced by Amoebas or AGNs. In this paper, the term "classic" for morphological operations refers to the use of non-adaptive structuring elements in such operations.

\section{Illustration}

In this section some examples of color morphological operations using the proposed CANs as structuring elements are shown. With comparison purposes, these operations have also been carried out on the same images with classic SEs, Amoebas and AGNs. Color spaces RGB, L*a*b* and HSL have been used. In this paper, the reference color $c_{0}$ in RGB has been valued $(255,255,255)$, and the component ordering $R \rightarrow G \rightarrow B$. In the case of the $\mathrm{L}^{*} \mathrm{a}^{*} \mathrm{~b}^{*}$ color space, $c_{0}=$ $(100,0,0)$ and the component ordering is $L * \rightarrow a * \rightarrow b *$. Finally these values in HSL have been $c_{0}=(0,0,1)$ (being all components valued in the interval $[0,1])$ and $L \rightarrow S \rightarrow H$. In all cases the original image has been used as pilot image (i.e., $f_{0}=f$ ).

In figure 2 these four methods are compared in the dilation operation processed in the $\mathrm{L}^{*} \mathrm{a} * \mathrm{~b} *$ color space. First of all, it is remarkable that the classic structuring elements drastically remove the small structures within the image, which also happens using the morphological amoebas, although in lesser extent. When CANs are used, these small structures and their borders are well preserved. Indeed, they preserve the borders of small details - such as the iris or the flowers under the eyes - better than the AGNs.

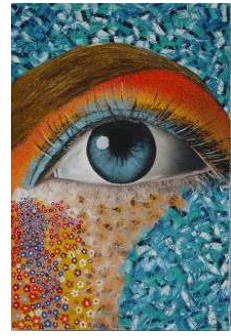

(a) Original Image

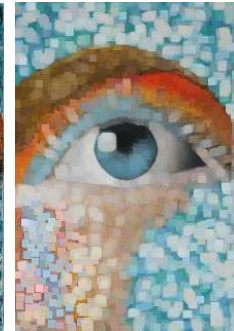

(b) Classic dilation (c) Amoeba dilation

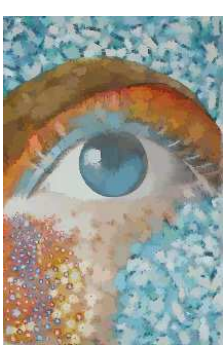

(d) AGN dilation

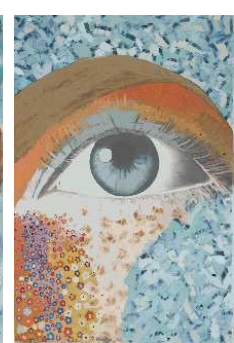

(e) CAN dilation
Fig. 2. Dilations of the image in (a) by means of square-shaped Structuring element with size 7 (b), morphological amoebas with radius 15 (c), AGNs with radius 60 (d) and CANs as ASEs with $m=15$ (e). The images are were processed in the $\mathrm{L} * \mathrm{a} * \mathrm{~b} *$ color space.

Figure 3 show the erosions by means of these four methods in the HSL color space. Once again, any of the adaptive methods preserves the inner structures and details within the 
image more than the classic one. By the way, CAN-based erosion preserves better the borders and details of the image than the Amoebas or AGN-based ones; for instance, CANs are more respectful with the small details of the image (i.e. the shape and borders of the small details of the original image are better preserved with the CAN-based morphological operations).

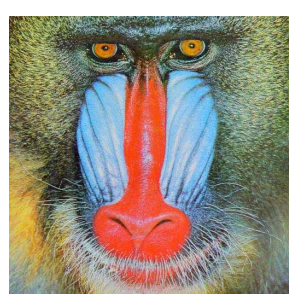

(a) Original Image

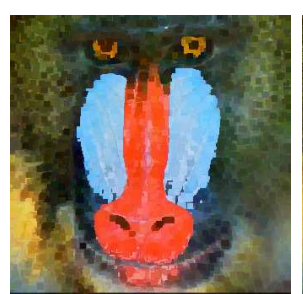

(b) Classic erosion

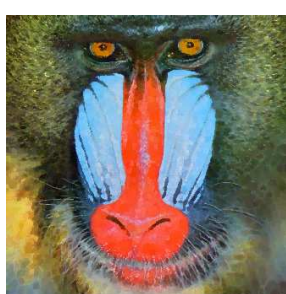

(c) Amoebas-based erosion

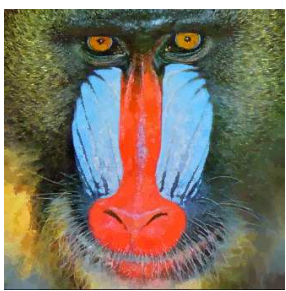

(d) AGN-based erosion

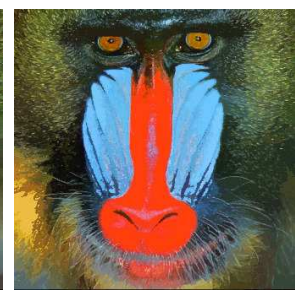

(e) CAN-based erosion
Fig. 3. Erosions of the image in (a) by means of square-shaped Structuring element with size 7 (b), morphological amoebas with radius 20 (c), AGNs with radius 80 (d) and CANs as ASEs with $m=30$ (e). The images are were processed in the HSL color space.

Figures 4 and 5 show the openings and closings processed within the color spaces RGB and $\mathrm{L}^{*} \mathrm{a} * \mathrm{~b} *$. In both figures, the opening and closing by reconstruction (figures 4(c) and 5(c) respectively) have also been computed for the sake of comparison. These operations do not preserve small details (details at the side of the mountain in the first case, or the horse and the man in the second). According to the comparison between the adaptive operations, the same remarks than in the case of the erosions and dilations can be made (which is specially noticeable in the example of the closing (figure 5).

\section{AppliCATION TO IMAGE CLASSIFICATION}

The proposed CAN-based color mathematical morphology has been applied to pixel-level classification in images of different textures. Concretely, each pixel has been described and each one has been assigned to a class by means of a neural network classifier. The next sections describe the images, descriptors, classification stage and, finally, the obtained results.

\section{A. Texture image database}

Five different classes have been classified, all of them extracted from the MIT Vision Texture (VisTex) database. Textures in this database are taken from different materials and divided into several categories. Some of them contain different

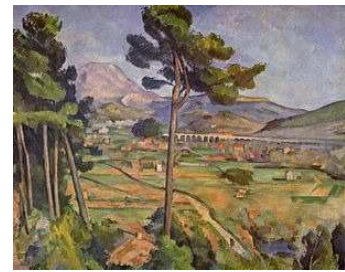

(a) Original Image

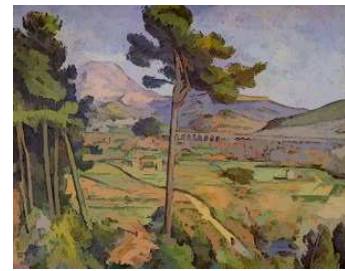

(c) Opening by reconstruction

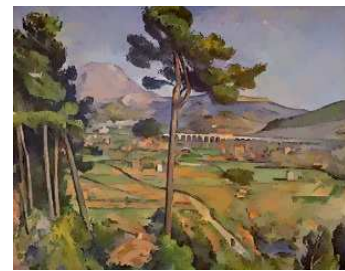

(e) AGN-based opening

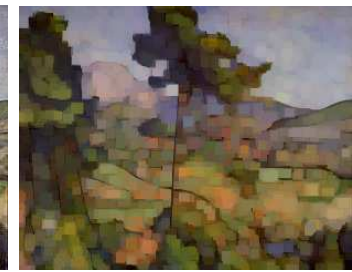

(b) Classic opening

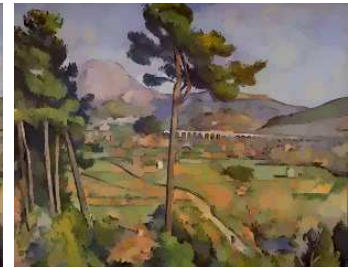

(d) Amoebas-based opening

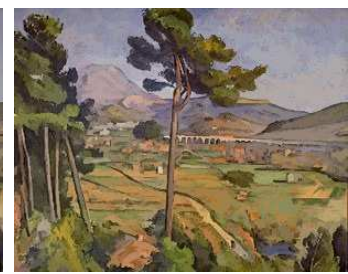

(f) CAN-based opening
Fig. 4. Openings of the image in (a) by means of square-shaped Structuring element with size 7 (b), opening by reconstruction with a square-shaped SE of size 5 (c), morphological amoebas with radius 30 (d), AGNs with radius 90 (e) and CANs as ASEs with $m=30$ (f). The images are were processed in the RGB color space.

types of textures, so they have been divided them into subcategories, as it was done in (González-Castro et al., 2012). An example of the sub-categories that have been used in this experiment is shown in figure 6 .

Each original image (whose spatial resolution is $512 \times 512$ pixels) was divided into 25 sub-images of $102 \times 102$ pixels each one which are not overlapped among themselves, as was made in (González-Castro et al., 2012). Thereafter, five images from each class were extracted randomly to be used in the experiment.

\section{B. Pixel-level description}

The descriptor of each pixel is formed by a concatenation of (1) its color components in the original image and (2) the color components of that point in successive dilations and erosions (i.e., the dilations and erosions using structuring elements of different sizes).

Four different descriptors have been assessed, changing the method (i.e., the kind of structuring element) to get the erosions and dilations. They were carried out using (i) a classic (i.e., non adaptive) square-shaped structuring element, (ii) CANs (section IV-B), (iii) morphological amoebas and (iv) AGNs (section IV-C). In all cases 20 dilations and 20 erosions 


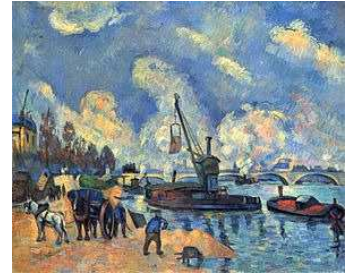

(a) Original Image

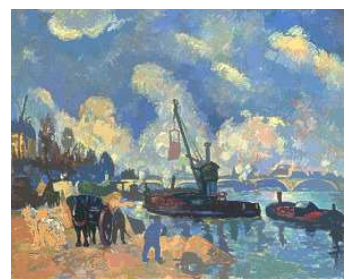

(c) Closing by reconstruction

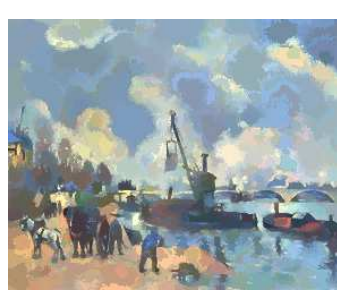

(e) AGN closing

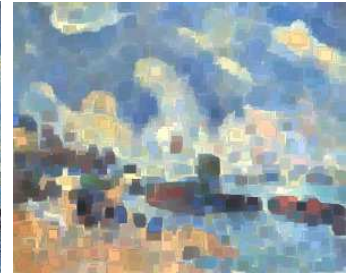

(b) Classic closing

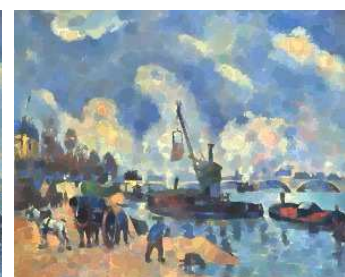

(d) Amoebas-based closing

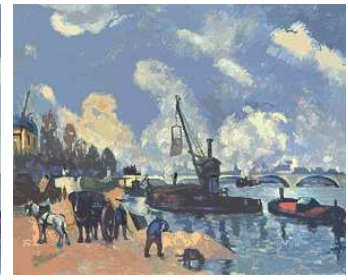

(f) CAN-based closing
Fig. 5. Closings of the image in (a) by means of square-shaped Structuring element with size 7 (b), closing by reconstruction with a square-shaped SE of size 5 (c), morphological amoebas with radius 15 (d), AGNs with radius 60 (e) CANs as ASEs with $m=15$ (f). The images are were processed in the $\mathrm{L}^{*} \mathrm{a} * \mathrm{~b} *$ color space

have been carried out. Therefore, each pixel is described by means of 123 features.

Therefore, the descriptor of each pixel $x$ in $D$ is:

$X_{n}(x)=\left[f(x), E_{p_{1}}^{f_{0}}(f)(x), D_{p_{1}}^{f_{0}}(f)(x), \ldots, E_{p_{n}}^{f_{0}}(f)(x), D_{p_{n}}^{f_{0}}(f)(x)\right]$

where $f(x)=\left\{f_{A}(x), f_{B}(x), f_{C}(x)\right\}$ (i.e., the values of the color components $A, B$ and $C$ at $x$ of the image $f$ ), and $E_{p_{i}}^{f_{0}}(f)(x)$ (resp. $D_{p_{i}}^{f_{0}}(f)(x)$ ) represents the values of the color components at $x$ of the erosion (resp. dilations) of $f$ with a structuring element (which may be classic, Amoeba, AGN or CAN) with size, radius or tolerance, respectively, $p_{i}$.

In this paper, the descriptor $X_{20}$ has been used to describe the pixels. However, the values of $p_{i}$ that have been taken are different for each method. Concretely, they have been set to:

- Classic: The sizes of the $\mathrm{SE}$ are $\left[p_{1}=1, p_{2}=\right.$ $\left.2, \ldots, p_{20}=20\right]$.

- CANs: The values for $m$ change from 5 to 100 in steps of 5: $\left[p_{1}=5, p_{2}=10, \ldots, p_{20}=100\right]$.

- Amoebas: The radii $r$, also change from 5 to 100 in steps of 5: $\left[p_{1}=5, p_{2}=10, \ldots, p_{20}=100\right]$.

- AGNs: The radii $r$ changes from 10 to 200 in steps of 10: $\left[p_{1}=10, p_{2}=20, \ldots, p_{20}=200\right]$.

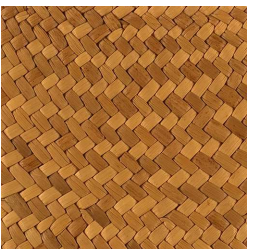

(a) Wicker $(\mathrm{FaWi})$

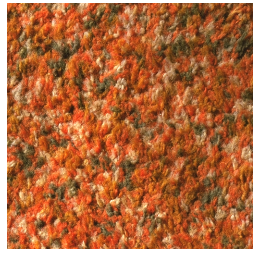

(b) Wool (FaWo)

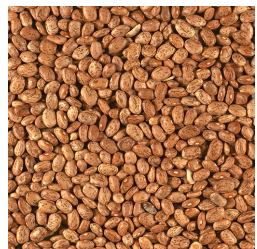

(c) Beans (FoBe)

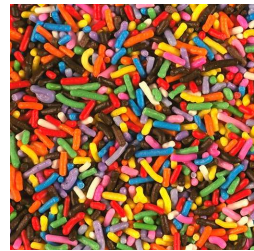

(d) Sweet chips (FoSw)

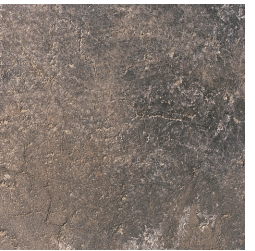

(e) Stone (Stone)
Fig. 6. Examples of used the VisTex sub-categories. The name that have been given to the class is written between brackets

These changes are due to the differences in size of the ANs in relation to their parameters parameters, as it is shown in figure 7. It is necessary to point out, however, that several values for the above mentioned parameters (in order to make the sizes to change) have been tried, and the results were comparable or worse to the ones shown in this paper.

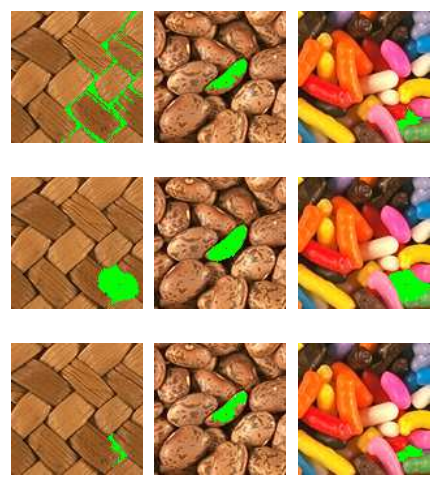

Fig. 7. Examples of CANs with $m=100$ (top), Morphological Amoebas with $r=100$ (middle) and AGNs with $r=200$ (bottom) of the classes FaBa (left), FoBe (center) and FoSw (right)

Since the color components of spatially contiguous pixels may be similar, as well as the colors of some dilations and erosions (e.g. the ones carried out with SEs of similar sizes), there might be a significant volume of highly correlated data. By the way, processing high dimensional data is computationally expensive and, due to this possible correlation, also inefficient. Hence, it is very important to extract uncorrelated components from the data, to be used as the final descriptors. In this application a Principal Component Analysis (PCA) has been employed to achieve this data dimensionality reduction in relation to the original descriptors (which had a dimension of 123). Therefore, the experiment was carried out fixing the number of principal components $(\mathrm{PC})$ to be extracted from the 
original descriptors from 1 to 20 , in order to assess the impact that the number of components might have on the classification results.

\section{Classification}

All these descriptors have been classified by means of a feed-forward Artificial Neural Network (ANN). In this experiment a network with one hidden layer and a logistic sigmoid activation function for the hidden and the output layers have been employed. Learning of the network was carried out with a momentum and adaptive learning rate algorithm. The number of neurons in the hidden layer have been set to 10 and the training was carried out during 400 cycles.

The training of the network has been carried out using the descriptors of pixels of 3 images randomly chosen per class (thus, a $60 \%$ of the data), and the pixels from the other 2 were used as test set. This process has been repeated 10 times, in order to avoid possible random effects. The presented results are an average of these 10 runs. Data were normalized so that they have mean zero and standard deviation one.

\section{Results}

The description has been carried out as it was explained in section V-B in the color spaces RGB, L*a*b* and HSL, extracting a number of principal components from the original descriptors varying between 1 and 20 and making the classifications for each one of them (therefore, 20 classifications per method at each color space have been carried out). In all cases the confusion matrices of the 5 classes have been computed, as well as the overall hit rate. For the sake of extension only the confusion matrices of the best method in each color space are shown.

In addition, figure 8 depicts a graphic example of the classification results with the different methods. Each pixel of the images in the first column has been assigned to a class (represented by a different color) by the Neural Network, using each of the assessed methods (columns 2 to 5). The description in this example was done in the RGB color space. In accordance with the overall results for this color space (Fig. 9(a)), CANs obtain the best classifications (being the FoSw class the one where there are more misclassified pixels), while AGNs obtain the worst (which is specially remarkable in the FaWo class).

Figure 9 plots the overall classification accuracy vs. the number of principal components extracted from the descriptors in the color spaces RGB, L*a*b* and HSL.

First of all, focusing on the results in the RGB color space, all descriptors have increasing hit rates up to a number of principal components between 6 and 9 (depending of the descriptor). Descriptors based on the proposed CANs obtain the best results in all scenarios between 3 and 5 principal components and from 8 on, where the accuracy is always higher than $94 \%$. The maximum hit rate $(96.16 \%)$ is reached when 15 principal components were extracted. It is specially remarkable the poor performance of the AGN, whose accuracies are always between about $83 \%$ and $89 \%$ (when extracting

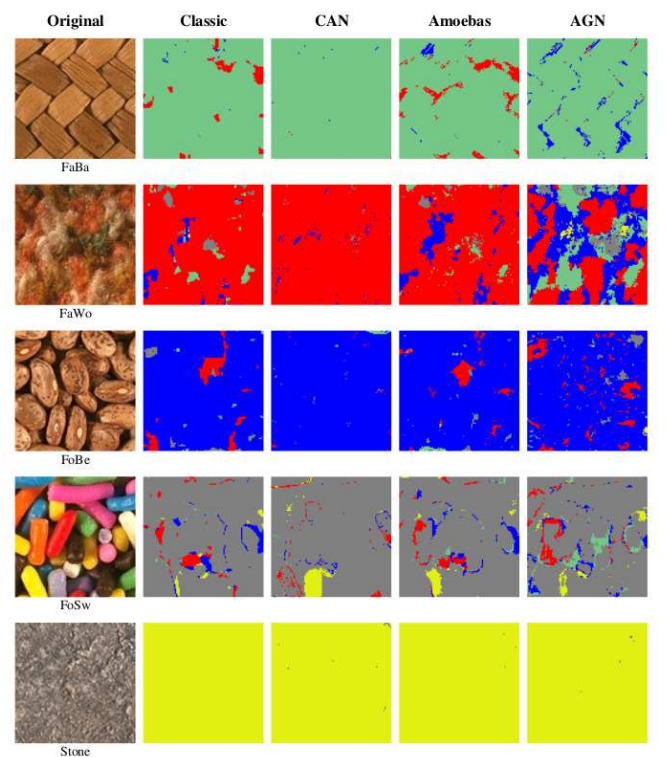

Fig. 8. Pixels from the image on the left, characterized by each descriptor, assigned by the classifier to a different class. The green, red, blue, gray and yellow colors stand for the FaBa, FaWo, FoBe, FoSw and Stone classes, respectively

more than $10 \mathrm{PCs}$ ). Focusing on the scenarios of 10 or more principal components, the amoeba-based descriptors and the descriptors based on the classic SEs are the second or third best ones depending on the number of principal components. However, regarding their maxima, amoebas perform better (94.1\% with 15 PCs, against $92.9 \%$ with 10 PCs).

In the case of the HSL color space, CANs also obtain the best performances in almost all scenarios from 4 PCs on, although the hit rates are lower. In this case, its best accuracy is obtained extracting 8 principal components (93.28\%). Once again, the worst results are obtained by AGNs. It is remarkable that in this color space and with more than 15 principal components, the classic SEs, CANs and Amoebas show very similar performances.

It is specially remarkable the poor performance of CANs in the $\mathrm{L}^{*} \mathrm{a} * \mathrm{~b} *$ color space, as well as the good one of AGNs and Amoebas (they are actually the best ones extracting 10 and 11 principal components with $96.57 \%$ and $96.71 \%$ respectively).

The confusion matrices of the classifier generated by the descriptor for the method which achieves the best accuracy on RGB, L*a*b* and HSL color spaces are shown in tables I, II and III, respectively. An initial conclusion upon observing them is that, in accordance with the results commented previously, the rates of misclassification upon the different classes are low, being the worst in the HSL color space. In accordance with what was seen in Fig. 8, the class FaWo is the one with less pixels well classified, in all color spaces (always less than $18.8 \%$ )

Finally, in order to assess the impact that the PCA has on the data the classification was also made with no previous extraction of principal components (i.e. on the vectors of 123 features). The results of these classifications are shown in table 


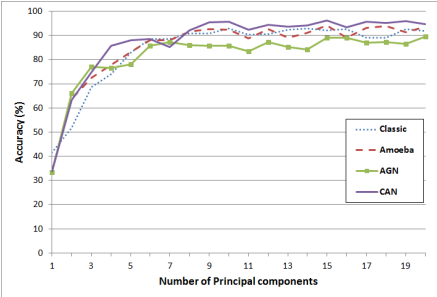

(a) RGB

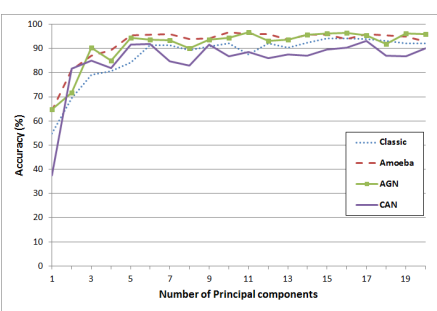

(b) $\mathrm{L} * \mathrm{a} * \mathrm{~b} *$

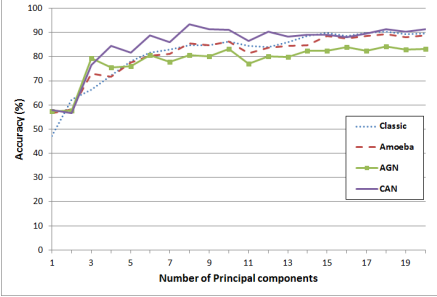

(c) HSL

Fig. 9. Curves of the hit rate vs. the number of principal components of the the assessed methods in the color spaces RGB (a), L*a*b* (b) and HSL (c).

TABLE I. CONFUSION MATRIX OF THE CLASSIFICATION OF 15 PRINCIPAL COMPONENTS EXTRACTED FROM THE CAN-BASED DESCRIPTORS IN THE RGB COLOR SPACE.

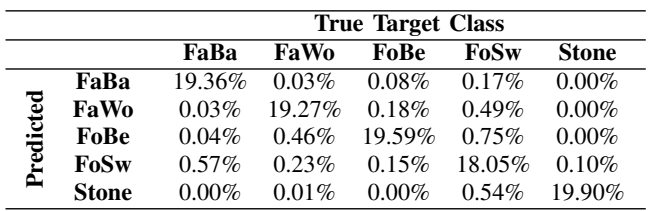

IV

In the RGB color space the best results are achieved by the CAN-based descriptors, although the accuracy is lower than when the PCA was done (which was around $96 \%$ with 15 PCs). In the case of $\mathrm{L}^{*} \mathrm{a}^{*} \mathrm{~b}^{*}$, the maximum accuracy is still reached by AGN-based descriptors, although this difference is

TABLE II. CONFUSION MATRIX OF THE CLASSIFICATION OF 11 PRINCIPAL COMPONENTS EXTRACTED FROM THE AGN-BASED DESCRIPTORS IN THE L*A*B* COLOR SPACE.

\begin{tabular}{ccccccc}
\hline & & \multicolumn{5}{c}{ True Target Class } \\
\hline & & FaBa & FaWo & FoBe & FoSw & Stone \\
\hline & FaBa & $19.75 \%$ & $0.23 \%$ & $0.15 \%$ & $0.06 \%$ & $0.00 \%$ \\
FaWo & $0.14 \%$ & $18.73 \%$ & $0.24 \%$ & $0.64 \%$ & $0.00 \%$ \\
FoBe & $0.07 \%$ & $0.30 \%$ & $19.47 \%$ & $0.26 \%$ & $0.00 \%$ \\
$:$ & FoSw & $0.04 \%$ & $0.69 \%$ & $0.10 \%$ & $18.79 \%$ & $0.03 \%$ \\
Stone & $0.00 \%$ & $0.05 \%$ & $0.04 \%$ & $0.25 \%$ & $19.97 \%$ \\
\hline
\end{tabular}

TABLE III. CONFUSION MATRIX OF THE CLASSIFICATION OF 8 PRINCIPAL COMPONENTS EXTRACTED FROM THE CAN-BASED DESCRIPTORS IN THE HSL COLOR SPACE.

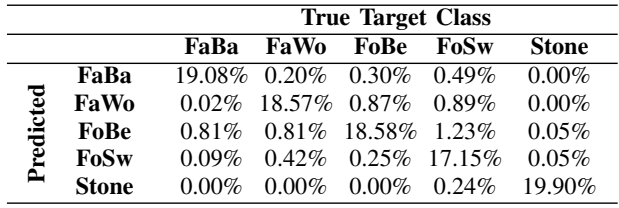

TABLE IV. ACCURACY (IN \%) OF THE CLASSIFICATIONS MADE ON THE DESCRIPTORS WITH NO PREVIOUS EXTRACTION OF PRINCIPAL COMPONENTS IN RGB, L*A*B* AND HSL.

\begin{tabular}{lcccc}
\hline & Classic & Amoebas & AGN & CAN \\
\hline RGB & 90.96 & 87.19 & 79.18 & $\mathbf{9 2 . 9 0}$ \\
L*a*b* & 90.82 & 93.43 & $\mathbf{9 5 . 2 7}$ & 79.23 \\
HSL & 92.59 & 91.72 & 84.78 & $\mathbf{9 6 . 1 2}$ \\
\hline
\end{tabular}

not so high $(95.27 \%$ against $96.7 \%$ when PCA was carried out). It is remarkable the performance in the HSL color space, where the accuracies are higher than when PCA was made on the data. This is very useful, as it allows to avoid the decision of how many principal components to take. This is maybe due to the higher correlation that the $\mathrm{R}, \mathrm{G}$ and $\mathrm{B}$ components have (Palus, 1998) compared with H, S and L ones.

\section{CONCLUSIONS AND FUTURE PERSPECTIVES}

In this work the GANIP approach (Debayle and Pinoli, 2006a), developed for gray-scale images, has been extended to color images. Therefore, the Color Adaptive Neighborhood (CAN) framework has been presented and its basis settled (i.e., comparing some measures to compute distances in color images). In addition, the way to apply the CANs to Mathematical Morphology has been shown. As a possible application of CAN-based mathematical morphology, a pixel description application based on it has also been shown.

In this context the performance of these morphological operators with classic SEs and other two kind of adaptive SEs found in the literature: morphological amoebas (Lerallut et al., 2007) and adaptive geodesic neighborhoods (Grazzini and Soille, 2009) have been compared in different color spaces both in morphological filtering operations and with pixel classification purposes. In the first case, it has been shown how CANs preserve the contrast and details of the images without damaging their color transitions. With respect to classification, even if the AGN-based description method performs quite well in $\mathrm{L}^{*} \mathrm{a} * \mathrm{~b} *$ (accuracy of $96.71 \%$ ), the CAN-based descriptors in HSL are very convenient, since they achieve a similar accuracy $(96.12 \%)$ without need to carry out a previous PCA, so it avoids the tuning of the number of principal components. These results show that CAN-based descriptors in the HSL color space might be useful, for example, for segmentation or remote sensing applications (Shi et al., 2013).

For future work, it is necessary to tackle the reasons for the differences in performance in the classification using the descriptors extracted from the RGB or HSL color spaces and the $\mathrm{L}^{*} \mathrm{a} * \mathrm{~b} *$ In addition, the development of CAN-based adaptive morphological operators in the CoLIP framework (Gouinaud 
et al., 2011) will be addressed, both as morphological filters and with description purposes.

\section{REFERENCES}

Angulo, J., 2007. Morphological colour operators in totally ordered lattices based on distances: Application to image filtering, enhancement and analysis. Computer Vision and Image Understanding 107, 56 - 73.

Aptoula, E., Lefvre, S., 2007. A comparative study on multivariate mathematical morphology. Pattern Recognition 40, $2914-2929$.

Aptoula, E., Lefvre, S., 2008. On lexicographical ordering in multivariate mathematical morphology. Pattern Recognition Letters 29, $109-118$.

Asano, A., Miyagawa, M., Fujio, M., 2003. Morphological texture analysis using optimization of structuring elements, in: Heidelberg, S.B. (Ed.), Geometry, Morphology, and Computational Imaging. Proceedings of the 11th International Workshop on Theoretical Foundations of Computer Vision, pp. 45-56.

Örsan Aytekin, lkay Ulusoy, 2011. Automatic segmentation of VHR images using type information of local structures acquired by mathematical morphology. Pattern Recognition Letters 32, 1618 - 1625.

Barnett, V., 1976. The ordering of multivariate data. Journal of the Royal Statistical Society. Series A 139, 318-354.

Bouaynaya, N., Charif-Chefchaouni, M., Schonfeld, D., 2006. Spatially variant morphological restoration and skeleton representation. IEEE Transactions on Image Processing 15, 3579-3591.

Bouaynaya, N., Charif-Chefchaouni, M., Schonfeld, D., 2008. Theoretical foundations of spatially-variant mathematical morphology part I: Binary images. Pattern Analysis and Machine Intelligence, IEEE Transactions on 30, 823-836.

Bouaynaya, N., Schonfeld, D., 2008. Theoretical foundations of spatially-variant mathematical morphology part II: Graylevel images. Pattern Analysis and Machine Intelligence, IEEE Transactions on 30, 837-850.

Bouraoui, B., Ronse, C., Baruthio, J., Passat, N., Germain, P., 2010. 3D segmentation of coronary arteries based on advanced mathematical morphology techniques. Computerized Medical Imaging and Graphics 34, 377 - 387.

van den Broek, E., Schouten, T., Kisters, P., 2008. Modeling human color categorization. Pattern Recognition Letters 29, $1136-1144$.

Busin, L., Vandenbroucke, N., Macaire, L., 2008. Color spaces and image segmentation, Elsevier. volume 151 of Advances in Imaging and Electron Physics, pp. 65 - 168.

CIE, 1995. Industrial Colour-Difference Evaluation. Technical Report 116. Commission Internationale de l'Éclairage CIE.

CIE, 2001. Improvement to Industrial Colour Difference Evaluation. Technical Report 142. Commission Internationale de l'Éclairage CIE.

Ćurić, V., Luengo Hendriks, C., Borgefors, G., 2012. Salience adaptive structuring elements. Selected Topics in Signal Processing, IEEE Journal of 6, 809-819.

Debayle, J., Pinoli, J.C., 2006a. General Adaptive Neighborhood Image Processing: Part I: Introduction and Theoretical
Aspects. Journal of Mathematical Imaging and Vision 25, 245-266.

Debayle, J., Pinoli, J.C., 2006b. General Adaptive Neighborhood Image Processing: Part II: Practical Application Examples. Journal of Mathematical Imaging and Vision 25, 267-284.

Debayle, J., Pinoli, J.C., 2014. Advances in Low-Level Color Image Processing. Springer. volume 11 of Lecture Notes in Computational Vision and Biomechanics. chapter Spatially Adaptive Color Image Processing. Chapter 6.

González-Castro, V., Alegre, E., García-Olalla, O., FernándezRobles, L., García-Ordás, M., 2012. Adaptive pattern spectrum image description using euclidean and geodesic distance without training for texture classification. Computer Vision, IET 6, 581-589.

Gouinaud, H., Gavet, Y., Debayle, J., Pinoli, J.C., 2011. Color correction in the framework of color logarithmic image processing, in: Image and Signal Processing and Analysis (ISPA), 2011 7th International Symposium on, Dubrovnik, Croatia. pp. 129-133.

Grazzini, J., Soille, P., 2009. Edge-preserving smoothing using a similarity measure in adaptive geodesic neighbourhoods. Pattern Recognition 42, 2306 - 2316.

Hanbury, A., Serra, J., 2001. Morphological operators on the unit circle. Image Processing, IEEE Transactions on 10, 1842-1850.

Haralick, R.M., Sternberg, S.R., Zhuang, X., 1987. Image analysis using mathematical morphology. IEEE Transactions on Pattern Analysis and Machine Intelligence 9, 532-550.

Huet, F., Mattioli, J., 1996. A textural analysis by mathematical morphology transformations: structural opening and top-hat, in: Proc. International Conference on Image Processing, pp. 49-52.

Joblove, G.H., Greenberg, D., 1978. Color spaces for computer graphics. SIGGRAPH Comput. Graph. 12, 20-25.

Landström, A., Thurley, M.J., 2013. Adaptive morphology using tensor-based elliptical structuring elements. Pattern Recognition Letters 34, 1416 - 1422.

Lerallut, R., Étienne Decencière, Meyer, F., 2007. Image filtering using morphological amoebas. Image and Vision Computing 25, $395-404$.

Matheron, G., 1975. Random Sets and Integral Geometry. John Wiley \& Sons.

Palus, H., 1998. Representations of colour images in different colour spaces, in: Sangwine, S., Horne, R. (Eds.), The Colour Image Processing Handbook. Springer US, pp. 6790.

Pinoli, J.C., Debayle, J., 2009. General adaptive neighborhood mathematical morphology, in: Image Processing (ICIP), 2009 16th IEEE International Conference on, pp. 22492252. Special session on adaptive morphology.

Priya, S., Kumar, T., Paul, V., 2011. A novel approach to fabric defect detection using digital image processing, in: Signal Processing, Communication, Computing and Networking Technologies (ICSCCN), 2011 International Conference on, pp. 228-232.

Serra, J., 1982. Image Analysis and Math. Morphology. 
Academic Press.

Shi, W., Miao, Z., Debayle, J., 2013. An integrated method for urban main-road centerline extraction from optical remotely sensed imagery. Geoscience and Remote Sensing, IEEE Transactions on PP, 1-14.

Shih, F.Y., Cheng, S., 2004. Adaptive mathematical morphology for edge linking. Information Sciences 167, 9 - 21.

Sternberg, S.R., 1986. Grayscale morphology. Computer Vision, Graphics, and Image Processing 35, 333-355.

Tuia, D., Pacifici, F., Kanevski, M., Emery, W., 2009. Classification of very high spatial resolution imagery using mathematical morphology and support vector machines. Geoscience and Remote Sensing, IEEE Transactions on 47, 3866-3879.

de Ves, E., Benavent, X., Ayala, G., Domingo, J., 2006. Selecting the structuring element for morphological texture classification. Pattern Analysis \& Applications 9, 48-57.

Yang, J., Liu, C., Zhang, L., 2010. Color space normalization: Enhancing the discriminating power of color spaces for face recognition. Pattern Recognition 43, 1454 - 1466. 